\title{
Space Densities of Cataclysmic Variables and Nova Recurrence Times
}

\author{
H.W. Duerbeck ${ }^{1,2}$, R. Covarrubias ${ }^{1}$ \\ 1 Astrophysics Group, P. Universidad Catolica de Chile, Santiago 22, Chile \\ 2 Astronomisches Institut der Universität Münster, D-48149 Münster, Germany
}

\begin{abstract}
Space densities of novae, dwarf novae and novalike stars are derived on the basis of the most recent catalogue of cataclysmic variables and improved values of absolute magnitudes. Assuming that these objects represent different stages of the same type of variable, changing over short-term cycles, we derive an average time interval of 10000 years between two subsequent nova eruptions. During this time, the cataclysmic variable remains almost 2000 years in the novalike stage and the remaining time in the dwarf nova stage, in which a total of nearly $3 \cdot 10^{-5} M_{\odot}$ is accreted on the white dwarf primary. This amount is comparable to the average mass of ejected nova shells.
\end{abstract}

\section{Introduction}

In 1983, one of the present authors determined the space densities of novae, dwarf novae, and related systems, and drew attention to the fact that for realistic outburst intervals, quiescent novae in the form of 'novalikes' appear to be underrepresented among cataclysmic variables (CVs) (Duerbeck 1984). Similar suggestions were voiced independently by Patterson (1984). This led to the revival and extension of the concept, first put forward by Vogt (1981), that novae spend their time between eruptions as novalike stars and as dwarf novae (see also Vogt 1989). Furthermore, the hibernation scenario was suggested by Shara et al. (1986), in which novae not only change into novalikes and dwarf novae, but may spend many thousands of years in a detached state, with a dramatic reduction of the accretion rate ('deep hibernation').

In the meantime, our knowledge of CVs and their physical properties has increased considerably. A recent catalogue of novae, dwarf novae, novalike stars and related systems is at hand (Downes and Shara 1993, henceforth D+S), and Warner (1987, henceforth W87) has determined improved absolute magnitudes for different types of CVs. It thus seemed worthwhile to check whether the 'deficit' of quiescent novae is still felt as strongly as in the past, and whether the 'deep hibernation' scenario is still needed. 


\section{Procedure}

From $D+S$, we take all dwarf nova (DN) and novalike (NL) systems, which are situated in a cylinder of $250 \mathrm{pc}$ radius, centered on the Sun and perpendicular to the galactic plane. Their $m_{\max }$ is obtained from the catalogue, an average $M_{\max }$ is assigned to each system (see below), interstellar extinction is taken into account with $A=1^{\mathrm{m}} / \mathrm{kpc}$, when the radius of vision is inside a slab of thickness $100 \mathrm{pc}$ above and below the galactic plane, and assumed to be $A=0$ otherwise, and the height $d_{z}$ above the plane is calculated.

Following W87, we assigned absolute magnitudes $M_{V}=+4.5$ to all dwarf novae at maximum, except those of SU UMa type, for which we assumed $M_{V}=$ +5.2 . Novalike stars show large scatter in their absolute magnitudes. We thus chose the average absolute magnitude in the sample of W87, i.e. $M_{V}=+4.1$. Distances were also calculated under the assumptions that $M_{V}$ differs by \pm 0.7 mag from this value.

The number of objects found in $z$-bins of $25 \mathrm{pc}$ were fitted with the simplex algorithm to the function

$$
\rho(z)=\rho_{0} \exp (-|z| / H)
$$

where $\rho_{0}$ is normalized to the space density per cubic parsec in the galactic plane, and $H$ is the scale height.

\section{Results}

Fitting the distribution of all dwarf novae by equation (1) yields:

$$
\rho(z)=(1.85 \pm 0.07) \cdot 10^{-6} \exp (-|z| /(98 \pm 5))\left[\mathrm{pc}^{-3}\right]
$$

For the U Gem/Z Cam-subclasses one obtains

$$
\rho(z)=(1.15 \pm 0.06) \cdot 10^{-6} \exp (-|z| /(96 \pm 6))\left[\mathrm{pc}^{-3}\right]
$$

For the SU UMa-subclass the result is

$$
\rho(z)=(0.70 \pm 0.03) \cdot 10^{-6} \exp (-|z| /(102 \pm 7))\left[\mathrm{pc}^{-3}\right]
$$

Within the error margins, both groups have the same space distribution, but the SU UMa-stars are roughly half as frequent as the U Gem/Z Cam-stars.

The space distribution of novalike stars was derived, assuming the absolute magnitudes given above. The exnova DQ Her was included in the sample since it fulfills all criteria for a NL in the solar neighbourhood, for which 'by chance' a nova eruption was observed. The following results for novalike stars are obtained, assuming $M_{V}(\max )=3.4$

$$
\rho(z)=(0.28 \pm 0.06) \cdot 10^{-6} \exp (-|z| /(97 \pm 32))\left[\mathrm{pc}^{-3}\right],
$$


assuming $M_{V}(\max )=4.1$

$$
\rho(z)=(0.42 \pm 0.06) \cdot 10^{-6} \exp (-|z| /(97 \pm 20))\left[\mathrm{pc}^{-3}\right]
$$

assuming $M_{V}(\max )=4.8$

$$
\rho(z)=(0.66 \pm 0.06) \cdot 10^{-6} \exp (-|z| /(70 \pm 9))\left[\mathrm{pc}^{-3}\right] .
$$

$M_{V}(\max )=4.8$ leads to a strong concentration of NL towards the galactic plane which appears unlikely. In the following, the result for $M_{V}(\max )=4.1$ is used.

For novae, we consider a cylinder with a radius $R_{x, y}=1500 \mathrm{pc}$ in the galactic plane. Since the compilation of the list of galactic novae in the solar vicinity (Duerbeck 1984), no bright nova has appeared within this cylinder, but many of the listed novae have now revised distances (Cohen 1985, W87). We take observed novae which erupted in the said volume during the 20 th century to derive the space density of nova eruptions per cubic parsec per year. The effective 'monitoring time', however, was set to $T_{\text {obs }}=70$ years, because novae may have been overlooked due to seasonal or other effects. With this assumption, the error of the result should not be larger than $25 \%$. We obtain the space/time density $\rho^{*}(z)$ :

$$
\rho^{*}(z)=(0.23 \pm 0.02) \cdot 10^{-9} \exp (-|z| /(120 \pm 24))\left[\mathrm{pc}^{-3} \mathrm{yr}^{-1}\right] .
$$

The above results show that the average scale height of all types of cataclysmic variables is about $110 \mathrm{pc}$. The number of objects in the solar neighbourhood, however, is too scarce to test whether they are a mixture of disk and bulge objects, as seems to be the case for novae (see Duerbeck 1990 and Della Valle at al. 1992).

\section{Discussion}

If we assume in a straightforward way that all dwarf novae and novalikes (except the highly magnetized polars, whose space density is possibly much lower), are manifestations of the same type of variable - a nova between two outbursts -, we can draw the following conclusions:

(1) The fact that DN are by a factor 4 more frequent than NL signifies that novae spend 4 times more time in the DN than in the NL stage.

(2) Assuming the space/time density of novae to be $0.23 \cdot 10^{-9} \mathrm{pc}^{-3} \mathrm{yr}^{-1}$, and the space density of DN plus NL $(1.85+0.42) \cdot 10^{-6} \mathrm{pc}^{-3}$, the average outburst interval $\Delta t$ of novae is (Duerbeck 1990):

$$
\Delta t=\frac{\rho^{*} \cdot T_{\mathrm{obs}}+\rho_{\mathrm{DN}}+\rho_{\mathrm{NL}}}{\rho^{*} \cdot T_{\mathrm{obs}}} \cdot T_{\mathrm{obs}} \approx \frac{\rho_{\mathrm{DN}}+\rho_{\mathrm{NL}}}{\rho^{*}}=\frac{2.27 \cdot 10^{-6}}{0.23 \cdot 10^{-9}}
$$

or $\Delta t \approx 10000$ years. If we assume that conclusion $(1)$ is valid, a nova between eruptions spends, on the average, 2000 years in the NL stage (including the socalled exnova-stage), and 8000 years in the DN stage. If accretion in the first stage is $10^{-8} M_{\odot} \mathrm{yr}^{-1}$, and in the second stage $10^{-9} M_{\odot} \mathrm{yr}^{-1}$, a total of about 
$3 \cdot 10^{-5} M_{\odot}$ is transferred, to be ejected in the subsequent explosion. This agrees with the average shell mass of novae, $3 \ldots 4 \cdot 10^{-5} M_{\odot}$, as determined by Cohen and Rosenthal (1983).

The above estimates can at best be taken as a guideline for further studies, because, e.g., the mass spectrum of accreting white dwarfs might have to be taken into account (e.g. Politano et al. 1990). Since the relation between critical mass and white dwarf mass seems to be still quite unclear, however, we refrain from discussing this problem here. A fainter stage ('deep hibernation') with a negligible accretion rate is possible, but not necessary, unless the 'average' mass transfer rate of about $3 \cdot 10^{-9} M_{\odot} \mathrm{yr}^{-1}$ is found to be too high on the basis of theoretical arguments.

Acknowledgements: We are grateful to Rolf Duemmler, Münster, for making his simplex function fitting program available to us, as well as to Ron Downes and Mike Shara (STScI) for the updated version of their catalogue. The stay of HWD with the Astrophysics Group of P.U.C. was made possible by a grant of the Deutscher Akademischer Austauschdienst.

\section{References}

Cohen J., 1985, ApJ 292, 90

Cohen J., Rosenthal A., 1983, ApJ 268, 689

Downes R.A., Shara M.M., 1993, PASP 105, 127 (D+S)

Della Valle M., Bianchini A., Livio M., Orio M., 1992, A\&A 266, 232

Duerbeck H.W., 1984, ApSS 99, 363

Duerbeck H.W., 1990, in IAU Coll. 122, Physics of Classical Novae, A. Cassatella and R. Viotti (eds.), Springer, Berlin, p. 34

Patterson J., 1984, ApJS 54, 443

Politano M., Livio M., Truran J.W., Webbink R.F., 1990, in IAU Coll. 122, Physics of Classical Novae, A. Cassatella and R. Viotti (eds.), Springer, Berlin, p. 386

Shara M.M., Livio M., Moffat A.F.J., Orio M., 1986, ApJ 311, 163

Vogt N., 1981, Mitt. Astr. Ges. 57, 79

Vogt N., 1989, in Classical Novae, M.F. Bode and A. Evans (eds.), Wiley, Chichester, p. 225

Warner B., 1987, MNRAS 227, 23 (W87) 